\title{
Linear Precoding Versus Linear Multiuser Detection in Downlink TDD-CDMA Systems
}

\author{
Inaki Berenguer, Xiaodong Wang, Manuel Donaire, Daryl Reynolds, and Anders Høst-Madsen
}

\begin{abstract}
In this paper, we compare two classes of linear interference suppression techniques for downlink TDD-CDMA systems, namely, linear multiuser detection methods (receiver processing) and linear precoding methods (transmitter processing). For the linear precoding schemes, we assume that the channel state information (CSI) is available only at the transmitter but not at the receiver (i.e., ultra simple receivers). We propose several precoding techniques and the corresponding power control algorithms. The performance metric used in the comparisons is the total power required at the transmitter to achieve a target SINR at the receiver. Our results reveal that in general multiuser detection and precoding offer similar performance; but in certain scenarios (e.g, low BER requirements or use of random spreading sequences), precoding can bring a substantial performance improvement. These results motivate the use of precoding techniques to reduce the complexity of the system and the mobile terminals (only a matched-filter to the own spreading sequence is required without CSI). Moreover, it is shown that the proposed chip-wise linear MMSE precoding method is optimal in the sense that it requires the minimum total transmitted power to meet a certain receiver SINR performance.
\end{abstract}

Index Terms-Downlink CDMA, linear multiuser detection, linear precoding, power control.

\section{INTRODUCTION}

$\mathbf{I}^{\mathbf{N}}$ $\mathrm{N}$ the uplink CDMA wireless systems, it is assumed that the base station has access to all users' channel state information (CSI) and spreading signatures; and multiuser detection (MUD) has been shown to be an effective way to combat interference and increase data throughput [12]. For the downlink, on the other hand, one can transfer the signal processing for interference suppression from the mobile receiver to the transmit base station by using precoding techniques. This is feasible if the base station has access to the CSI of all active mobile units, e.g., in time-division duplex (TDD) systems where the base station can exploit the channel reciprocity if the time difference between uplink and downlink transmission is shorter than the channel coherence time, or by using channel prediction techniques [1]. The

Manuscript received April 14, 2005; revised April 5, 2006 and August 28, 2006; accepted October 18, 2006. The associate editor coordinating the review of this letter and approving it for publication was D. Gesbert.

I. Berenguer is with the Engineering Dept., University of Cambridge, Cambridge, UK (e-mail: ib@ee.columbia.edu).

$\mathrm{X}$. Wang is with the Dept. of Electrical Engineering, Columbia University, New York, NY 10027 USA (e-mail: wangx@ee.columbia.ed).

M. Donaire is with the Dept. of Applied Mathematics \& Theoretical Physics, University of Cambridge, Cambridge, UK.

D. Reynolds is with the Dept. of Computer Science and Electrical Engineering, West Virginia University, 837 Engineering Sciences Building, PO Box 610, Morgantown, WV 26506 USA (e-mail: reynolds@csee.wvu.edu).

A. Høst-Madsen is with the Dept. of Electrical Engineering, University of Hawaii, Honolulu, HI 96817 USA.

Digital Object Identifier 10.1109/TWC.2006.xxxxx. simplest precoding method is pre-RAKE [2], which mitigates the multipath interference without considering the multiuser interference (MUI). Linear precoding techniques to remove the MUI and multipath interference were proposed in [13]. Nonlinear precoding techniques have been shown to offer superior performance although they complicate the receiver and the transmitter, since a modulo operation (which in general depends on the CSI) has to be implemented at both sides of the communication link [3], [9], [14]. Note that most work on linear precoding assumes that each user implements a RAKE receiver and hence assumes the knowledge of CSI at the receiver [13], [14].

In this paper, we consider linear precoders with ultra-simple receivers, i.e., only a fixed matched-filter to the spreading sequence without CSI. We propose several bit-wise and chipwise linear precoders and the corresponding power control algorithms to meet certain performance at the receiver. We also consider the performance comparisons between linear precoding and linear MUD. The comparison metric is the total required power at the transmitter to achieve a minimum SINR requirement at each of the receivers. Our results show that linear precoding offers similar performance as linear MUD in most cases; but in some specific cases (e.g., low BER requirements, heavily loaded systems, or use of random spreading sequences), linear precoding can be more effective. Moreover, the proposed linear precoding techniques with only the matched-filter (to the spreading sequence) at the receiver can outperform the linear precoder with a RAKE receiver (i.e., with CSI at the receiver) proposed in [13]. These results motivate the use of linear precoding techniques in the downlink of TDD-CDMA systems. Among the advantages of using linear precoding we have:

- Receiver terminals are limited to a fixed matched-filter to the own spreading sequence. This translates into a power consumption reduction and decrease in price of the terminals. Note that variations in channel conditions and number of active users in the network do not affect the receiver operations.

- A reduced amount of control data is required in the precoding solution. The reason is that in MUD, every user requires to know the own channel response plus the spreading sequences of all other active users in the network. Moreover, mobile units do not need to be informed when users are added to (or removed from) the network.

- Power control is easy to implement with linear precoding since the receiver has information about the quality of each link and it does not require extra feedback information. Note that MUD requires a feedback link to find the 
power loading value assigned to each user.

- Opportunistic user scheduling based on the knowledge of CSI [7], [8] can be implemented jointly with linear precoding to increase the system throughput.

The remainder of this paper is organized as follows. In Section 2 we briefly summarize two well-known linear MUD methods and the corresponding power control algorithms. In Section 3 we propose several forms of linear precoding schemes and discuss their properties. In Section 4 we present simulation comparisons between linear MUD and linear precoding. Finally, Section 5 concludes the paper.

\section{LINEAR MUD METHODS}

In this section, we give a short overview of two basic MUD solutions. We consider a $K$-user discrete-time synchronous multipath CDMA system. Define $b_{k}[i]$ from a constellation $\mathcal{A}$ as the symbol of the $k$-th user transmitted during the $i$-th symbol interval with $\mathbb{E}\left\{\left|b_{k}[i]\right|^{2}\right\}=1$ and $\boldsymbol{b}[i]=\left[b_{1}[i], \ldots, b_{K}[i]\right]^{T}$. Denote $N$ as the spreading factor and $\boldsymbol{s}_{k}=\left[s_{k, 1}, \ldots, s_{k, N}\right]^{T}$ as the normalized spreading waveform of the $k$-th user. Then, the signal transmitted from the base station during the $i$-th symbol interval can be written as $\boldsymbol{p}[i]=\boldsymbol{S} \boldsymbol{A} \boldsymbol{b}[i]$, where $\boldsymbol{S}=\left[\boldsymbol{s}_{1}, \boldsymbol{s}_{2}, \ldots, \boldsymbol{s}_{K}\right]$ is the matrix of spreading waveforms; and $\boldsymbol{A}=\operatorname{diag}\left(A_{1}, \ldots, A_{K}\right)$ contains the user signal amplitudes. The vector $\boldsymbol{p}[i]$ is passed through a parallel-to-serial converter and transmitted over the multipath channel. The path delays are assumed to be an integral number of chip periods. Denote the multipath channel seen by the $k$-th user as $\boldsymbol{f}_{k}=$ $\left[f_{k, 1}, f_{k, 2}, \ldots, f_{k, L}\right]^{T}$, where $L$ is the number of resolvable paths and $f_{k, l}$ is the complex fading gain corresponding to the $l$-th path of the $k$-th user. We assume that $L<N$. At the $k$-th user's receiver, the $N \times 1$ received signal during $N$ consecutive chip intervals corresponding to $\boldsymbol{b}[i]$ is given by

$$
\begin{aligned}
\boldsymbol{r}_{k}[i]= & \underbrace{\boldsymbol{F}{ }_{k} \boldsymbol{S}}_{\boldsymbol{H}_{k}} \boldsymbol{A} \boldsymbol{b}[i]+\boldsymbol{n}_{k}[i] \quad \text { with } \\
b F_{k}= & {\left[\begin{array}{ccccc}
f_{k, 1} & 0 & \ldots & \ldots & 0 \\
\vdots & \ddots & \ddots & & \vdots \\
f_{k, L} & \ddots & f_{k, 1} & \ddots & \\
0 & \ddots & & \ddots & 0 \\
0 & \cdots & f_{k, L} & \cdots & f_{k, 1}
\end{array}\right]_{N \times N} }
\end{aligned}
$$

where $\boldsymbol{r}_{k}[i]=\left[r_{k, 1}[i], \ldots, r_{k, N}[i]\right]^{T}$ is the received signal, $\boldsymbol{n}_{k}[i] \sim \mathcal{N}_{c}\left(\mathbf{0}, \sigma^{2} \boldsymbol{I}_{N}\right)$ is the complex white Gaussian noise vector at the $k$-th receiver, and $\boldsymbol{H}_{k}=\boldsymbol{F}_{k} \boldsymbol{S}$. Notice that we have assumed that ISI can be ignored either by being truncated or by inserting a guard interval. At the $k$-th receiver, a linear detector to recuperate the signal $b_{k}[i]$ can be represented by an $N$-dimensional vector $\boldsymbol{w}_{k} \in \mathbb{C}^{N}$, which is correlated with the received signal $\boldsymbol{r}_{k}[i]$ in (1) to obtain $z_{k}[i]=\boldsymbol{w}_{k}^{H} \boldsymbol{r}_{k}[i]$, and the $k$-th mobile unit makes a decision $\hat{b}_{k}[i]=\mathcal{Q}\left(z_{k}[i]\right)$, where $\mathcal{Q}$ rounds to the closest point in the constellation.

Linear Decorrelating Detector: The decorrelating detector completely eliminates the multiuser interference (MUI) and interchip interference (ICI), at the expense of enhancing the noise. The linear decorrelating detector for user $k$ is given by [12]

$$
\boldsymbol{w}_{k}=\boldsymbol{H}_{k}^{\dagger H} \boldsymbol{e}_{k}=\boldsymbol{H}_{k}\left(\boldsymbol{H}_{k}^{H} \boldsymbol{H}_{k}\right)^{-1} \boldsymbol{e}_{k}
$$

where $\boldsymbol{e}_{k}$ denotes a $K$-dimensional vector with all entries zeros, except for the $k$-th entry, which is 1 . The output of this detector is given by

$$
\begin{array}{r}
z_{k}[i]=\boldsymbol{w}_{k}^{H} \boldsymbol{r}_{k}[i]=A_{k} b_{k}[i]+\boldsymbol{w}_{k}^{H} \boldsymbol{n}_{k}[i] \\
\Longrightarrow \operatorname{SINR}_{k}=\frac{A_{k}^{2}}{\sigma^{2}\left\|\boldsymbol{w}_{k}\right\|^{2}},
\end{array}
$$

where $\mathrm{SINR}_{k}$ is the signal-to-interference-plus-noise ratio for the $k$-th user. Suppose that the SINR requirement for user $k$ is such that $\operatorname{SINR}_{k} \geq \gamma_{k}$, where $\gamma_{k}$ is the minimum acceptable SINR value for user $k$. Hence we have $A_{k}^{2}=\sigma^{2} \gamma_{k}\left\|\boldsymbol{w}_{k}\right\|^{2}$. And the total required transmit power is given by

$$
P_{T}=\sum_{k=1}^{K} A_{k}^{2}=\sum_{k=1}^{K} \sigma^{2} \gamma_{k} \boldsymbol{e}_{k}^{H}\left(\boldsymbol{S}^{H} \boldsymbol{F}_{k}^{H} \boldsymbol{F}_{k} \boldsymbol{S}\right)^{-H} \boldsymbol{e}_{k}
$$

Linear MMSE Detector: The linear MMSE detector for user $k$ is given by [12]

$$
\begin{aligned}
\boldsymbol{w}_{k} & =\arg \min _{\boldsymbol{w}_{k} \in \mathbb{C}^{N}} \mathbb{E}\left\{\left|b_{k}[i]-\boldsymbol{w}_{k}^{H} \boldsymbol{r}_{k}[i]\right|^{2}\right\} \\
& =A_{k}\left(\boldsymbol{H}_{k} \boldsymbol{A}^{2} \boldsymbol{H}_{k}^{H}+\sigma^{2} \boldsymbol{I}_{N}\right)^{-1} \boldsymbol{H}_{k} \boldsymbol{e}_{k} .
\end{aligned}
$$

The SINR for this detector is given by

$$
\operatorname{SINR}_{k}=\frac{A_{k}^{2}\left\|\boldsymbol{w}_{k}^{H} \boldsymbol{H}_{k} \boldsymbol{e}_{k}\right\|^{2}}{\sum_{j \neq k} A_{j}^{2}\left\|\boldsymbol{w}_{k}^{H} \boldsymbol{H}_{k} \boldsymbol{e}_{j}\right\|^{2}+\sigma^{2}\left\|\boldsymbol{w}_{k}\right\|^{2}} .
$$

We seek to minimize the total power $P_{T}$ such that $\operatorname{SINR}_{k} \geq$ $\gamma_{k}$. The iterative power control algorithm for linear MMSE MUD proposed in [11] can be extended to the downlink scenario, as we show in Algorithm 1 . At the $(n+1)$-th iteration, the MMSE filter $\boldsymbol{w}_{k}(n+1)$ is constructed using the current power matrix $\boldsymbol{A}(n)$. Then, the power matrix $\boldsymbol{A}(n+1)$ is updated using the new filter coefficients $\boldsymbol{w}_{k}(n+1)$.

\section{LINEAR PRECODING SCHEMES}

In this section we consider different approaches to implement linear precoding assuming that the transmitter has perfect CSI.

\section{A. Bit-Wise Linear Precoding}

We assume that each mobile unit employs only a filter matched to its own spreading sequence, and it does not need to know other users' spreading sequences or to estimate the channel. Denote the symbol by symbol bit-wise precoding operation as $\boldsymbol{x}[i]=\boldsymbol{M}_{b} \boldsymbol{A} \boldsymbol{b}[i]$, where $\boldsymbol{x}[i]$ is the precoded symbol vector and $\boldsymbol{M}_{b} \in \mathbb{C}^{K \times K}$ is the bit-wise linear precoding matrix. Then, after spreading the precoded data, the signal transmitted from the base station during the $i$-th symbol interval can be written as $\boldsymbol{p}[i]=\boldsymbol{S} \boldsymbol{x}[i]=\boldsymbol{S} \boldsymbol{M}_{b} \boldsymbol{A} \boldsymbol{b}[i]$. The vector $\boldsymbol{p}[i]$ is passed through a parallel-to-serial converter and transmitted through the channel. The signal received by the $k$-th user is then given by

$$
\boldsymbol{r}_{k}[i]=\boldsymbol{F}_{k} \boldsymbol{S} \boldsymbol{M}_{b} \boldsymbol{A} \boldsymbol{b}[i]+\boldsymbol{n}_{k}[i],
$$




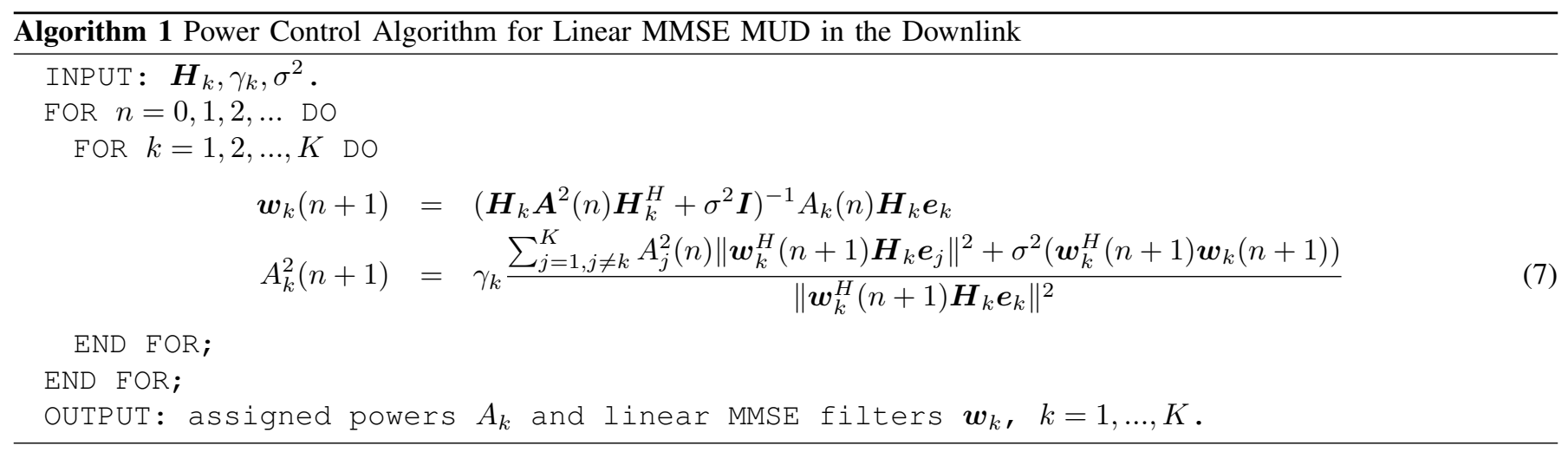

where $\boldsymbol{F}_{k}$ is given in (1). Then the corresponding matched filter $\boldsymbol{s}_{k}$ is applied to $\boldsymbol{r}_{k}[i]$. Stacking the outputs of the $K$ matched-filters we obtain

$\underbrace{\left[\begin{array}{c}\boldsymbol{s}_{1}^{H} \boldsymbol{r}_{1}[i] \\ \boldsymbol{s}_{2}^{H} \boldsymbol{r}_{2}[i] \\ \vdots \\ \boldsymbol{s}_{K}^{H} \boldsymbol{r}_{K}[i]\end{array}\right]}_{\boldsymbol{y}[i]}=\underbrace{\left[\begin{array}{c}\boldsymbol{s}_{1}^{H} \boldsymbol{F}_{1} \boldsymbol{S} \\ \boldsymbol{s}_{1}^{H} \boldsymbol{F}_{2} \boldsymbol{S} \\ \vdots \\ \boldsymbol{s}_{K}^{H} \boldsymbol{F}_{K} \boldsymbol{S}\end{array}\right]}_{\boldsymbol{H}_{b}} \boldsymbol{M}_{b} \boldsymbol{A b}[i]+\underbrace{\left[\begin{array}{c}\boldsymbol{s}_{1}^{H} \boldsymbol{n}_{1}[i] \\ \boldsymbol{s}_{2}^{H} \boldsymbol{n}_{2}[i] \\ \vdots \\ \boldsymbol{s}_{K}^{H} \boldsymbol{n}_{K}[i]\end{array}\right]}_{\boldsymbol{v}[i]}$.

The $k$-th receiver makes a decision $\hat{b}_{k}[i]=\mathcal{Q}\left(y_{k}[i]\right)$. Therefore the precoder design problem involves designing the precoding matrix $\boldsymbol{M}_{b}$ such that $\boldsymbol{r}[i]$ is as close to $\boldsymbol{b}[i]$ as possible.

Bit-wise Linear MMSE Precoder: Assuming that the spreading sequences are normalized, the linear MMSE precoder chooses the precoding matrix $\boldsymbol{M}_{b}$ to minimize $\mathbb{E}\{\| \boldsymbol{b}-$ $\left.\boldsymbol{y} \|^{2}\right\}$, and is given by $\boldsymbol{M}_{b}=\beta \boldsymbol{H}_{b}^{-1}$ [10], [13], where $\beta=\sqrt{\frac{P_{T}}{\operatorname{tr}\left(\boldsymbol{S} \boldsymbol{H}_{b}^{-1} \boldsymbol{A}^{2} \boldsymbol{H}_{b}^{-H} \boldsymbol{S}^{H}\right)}}$ is introduced to guarantee the power constraint at the transmitter $P_{T}$. Note that such a linear MMSE precoder also zero-forces the interference. When the constraint becomes the minimum SINR requirement at each receiver $\gamma_{k}$ instead of the available power $P_{T}$, we obtain the unconstrained precoding solution $\boldsymbol{M}_{b}=\boldsymbol{H}_{b}^{-1}$ with $\mathrm{SINR}_{k}=$ $\frac{A_{k}^{2}}{\sigma^{2}}$; and therefore the power assigned to the $k$-th user becomes $A_{k}^{2}=\sigma^{2} \gamma_{k}$. Then the total power required at the transmitter becomes $P_{T}=\mathbb{E}\left\{\left\|\boldsymbol{S} \boldsymbol{M}_{b} \boldsymbol{A} \boldsymbol{b}[i]\right\|^{2}\right\}=\operatorname{tr}\left(\boldsymbol{S} \boldsymbol{M}_{b} \boldsymbol{A}^{2} \boldsymbol{M}_{b}^{H} \boldsymbol{S}^{H}\right)$.

Bit-wise Wiener Precoder: The bit-wise Wiener precoder is proposed in [5], [6] as the matrix $M_{b}$ and constant $\beta$ that minimize $\mathbb{E}\left\{\left\|\boldsymbol{b}[i]-\beta^{-1} \boldsymbol{y}[i]\right\|^{2}\right\}$, subject to $\mathbb{E}\left\{\left\|\boldsymbol{M}_{b} \boldsymbol{A} \boldsymbol{b}[i]\right\|^{2}\right\}=$ $P_{T}$. Given the total transmit power $P_{T}$, the Wiener precoder is given by

$$
\begin{gathered}
\boldsymbol{M}_{b}=\beta \boldsymbol{F}^{-1} \boldsymbol{H}_{b}^{H}, \quad \text { with } \beta=\sqrt{\frac{P_{T}}{\operatorname{tr}\left(\boldsymbol{F}^{-2} \boldsymbol{H}_{b}^{H} \boldsymbol{A}^{2} \boldsymbol{H}_{b}\right)}} \\
\text { and } \boldsymbol{F}=\boldsymbol{H}_{b}^{H} \boldsymbol{H}_{b}+\frac{K \sigma^{2}}{P_{T}} \boldsymbol{I}_{N} .
\end{gathered}
$$

Optimal Transmit Spreading Sequences: Besides optimizing the precoding matrix $\boldsymbol{M}_{b}$ for a given channel realization, we can also optimize the transmit spreading sequences. Denote $s_{1}, \ldots, s_{K}$ as the fixed spreading sequences used at the mobile units (i.e., the matched -filters) and $\tilde{\boldsymbol{s}}_{1}, \ldots, \tilde{\boldsymbol{s}}_{K}$ as the optimized spreading sequences used at the transmitter. Denote $\tilde{\boldsymbol{S}}=$ $\left[\tilde{\boldsymbol{s}}_{1}, \ldots, \tilde{\boldsymbol{s}}_{K}\right]$. Similarly to (9), the received signal can be written as

$$
\underbrace{\left[\begin{array}{c}
\boldsymbol{s}_{1}^{H} \boldsymbol{r}_{1}[i] \\
\boldsymbol{s}_{2}^{H} \boldsymbol{r}_{2}[i] \\
\vdots \\
\boldsymbol{s}_{K}^{H} \boldsymbol{r}_{K}[i]
\end{array}\right]}_{\boldsymbol{y}[i]}=\underbrace{\left[\begin{array}{c}
\boldsymbol{s}_{1}^{H} \boldsymbol{F}_{1} \\
\boldsymbol{s}_{1}^{H} \boldsymbol{F}_{2} \\
\vdots \\
\boldsymbol{s}_{K}^{H} \boldsymbol{F}_{K}
\end{array}\right]}_{\boldsymbol{H}_{c}} \tilde{\boldsymbol{S}} \boldsymbol{M}_{b} \boldsymbol{A} \boldsymbol{b}[i]+\underbrace{\left[\begin{array}{c}
\boldsymbol{s}_{1}^{H} \boldsymbol{n}_{1}[i] \\
\boldsymbol{s}_{2}^{H} \boldsymbol{n}_{2}[i] \\
\vdots \\
\boldsymbol{s}_{K}^{H} \boldsymbol{n}_{K}[i]
\end{array}\right]}_{\boldsymbol{v}[i]} .
$$

Following [10], it can be easily shown that the linear MMSE precoding matrix is given by $\boldsymbol{M}_{b}=\left(\boldsymbol{H}_{c} \tilde{\boldsymbol{S}}\right)^{-1}$, and $A_{k}^{2}=$ $\sigma^{2} \gamma_{k}, k=1, \ldots, K$. Next we show that for any given propagation channel $\boldsymbol{F}_{1}, \ldots, \boldsymbol{F}_{K}$, original spreading sequences $\boldsymbol{S}$, and minimum SINR requirements, we can explicitly find the optimal spreading matrix $\tilde{\boldsymbol{S}}^{*} \in \mathbb{C}^{N \times K}$ such that the total transmit power $P_{T}$ is minimized. Assume that the $K \times N$ matrix $\boldsymbol{H}_{c}$ has rank $K$, where $N \geq K$. Define the SVD $\boldsymbol{H}_{c}=\boldsymbol{U}_{c} \boldsymbol{\Sigma}_{c} \boldsymbol{V}_{c}^{H}$, where $\boldsymbol{U}_{c}$ is a $K \times K$ unitary matrix, $\boldsymbol{V}_{c}^{H}$ is an $N \times N$ unitary matrix and $\boldsymbol{\Sigma}_{c}$ is a $K \times N$ diagonal matrix with $\left[\boldsymbol{\Sigma}_{c}\right]_{i, i}=\lambda_{c, i}$ being the positive square root of the $i$-th eigenvalue of $\boldsymbol{H}_{c} \boldsymbol{H}_{c}^{H}$.

Proposition 1: Given the channels $\boldsymbol{F}_{1}, \ldots, \boldsymbol{F}_{K}$, the receiver matched-filters $s_{1}, \ldots, s_{K}$, and the target SINR $\gamma_{1}, \ldots, \gamma_{K}$ of all users, by optimizing the transmit spreading matrix $\tilde{\boldsymbol{S}}$ used in the bit-wise linear MMSE precoder, the minimum achievable transmit power is given by

$$
P_{T}^{*}=\min _{\tilde{\boldsymbol{S}} \in \mathbb{C}^{N \times K}} \operatorname{tr}\left(\tilde{\boldsymbol{S}} \boldsymbol{M}_{b} \boldsymbol{A}^{2} \boldsymbol{M}_{b}^{H} \tilde{\boldsymbol{S}}^{H}\right)=\sum_{k=1}^{K} A_{k}^{2} \lambda_{c, k}^{-2},
$$

where $A_{k}^{2}=\sigma^{2} \gamma_{k}, k=1, \ldots, K$ are the assigned powers. One solution to the optimization problem in (12) (i.e., the optimal transmit spreading matrix) is given by the $N \times K$ matrix $\tilde{\boldsymbol{S}}^{*}=\boldsymbol{H}_{c}^{H}$.

Proof: Note that $\boldsymbol{M}_{b}=\left(\boldsymbol{H}_{c} \tilde{\boldsymbol{S}}\right)^{-1}$ and therefore the transmitted vector is given by $\boldsymbol{p}[i]=\tilde{\boldsymbol{S}} \boldsymbol{M}_{b} \boldsymbol{A} \boldsymbol{b}[i]=\tilde{\boldsymbol{S}}\left(\boldsymbol{H}_{c} \tilde{\boldsymbol{S}}\right)^{-1} \boldsymbol{A} \boldsymbol{b}[i]$. Denote the SVDs of $\boldsymbol{H}_{c}$ and $\tilde{\boldsymbol{S}}$ by $\boldsymbol{H}_{c}=\boldsymbol{U}_{c} \boldsymbol{\Sigma}_{c} \boldsymbol{V}_{c}^{H}$ and $\tilde{\boldsymbol{S}}=\boldsymbol{U}_{\tilde{s}} \boldsymbol{\Sigma}_{\tilde{s}} \boldsymbol{V}_{\tilde{s}}^{H}$, respectively. Then the total transmit power is shown in (13), where $\boldsymbol{T}=\boldsymbol{\Sigma}_{c} \boldsymbol{V}_{c}^{H} \boldsymbol{U}_{s} \boldsymbol{\Sigma}_{s}$ is a $K \times K$ matrix; $\boldsymbol{\Sigma}_{\tilde{s}}^{2}=\boldsymbol{\Sigma}_{\tilde{s}}^{H} \boldsymbol{\Sigma}_{\tilde{s}}$ is a $K \times K$ diagonal matrix; and we used the fact that $\boldsymbol{U}_{\tilde{s}}, \boldsymbol{U}_{c}, \boldsymbol{V}_{c}$ and $\boldsymbol{V}_{s}$ are unitary.

Consider $\boldsymbol{T}$ expressed in terms of the matrices obtained with the thin SVD [4], $\boldsymbol{T}=\boldsymbol{\Sigma}_{c}^{(t)} \boldsymbol{C} \boldsymbol{\Sigma}_{\tilde{s}}^{(t)}$, where $\boldsymbol{\Sigma}_{c}^{(t)}$ and $\boldsymbol{\Sigma}_{\tilde{s}}^{(t)}$ are the $K$-th leading submatrix of $\boldsymbol{\Sigma}_{c}$ and $\boldsymbol{\Sigma}_{\tilde{s}}$, respectively; and $\boldsymbol{C}=\boldsymbol{V}_{c}^{(t) H} \boldsymbol{U}_{s}^{(t)}$ is a $K \times K$ matrix (where $\boldsymbol{V}_{c}^{(t)}$ and $\boldsymbol{U}_{s}^{(t)}$ denote the matrices consisting of the first $K$ columns of $\boldsymbol{U}_{\tilde{s}}$ and $\boldsymbol{V}_{c}$, respectively). Denoting $\left\{\boldsymbol{v}_{c, 1}, \ldots, \boldsymbol{v}_{c, K}\right\}$ and 


$$
\begin{aligned}
P_{T} & =\mathbb{E}\left\{\boldsymbol{p}^{H}[i] \boldsymbol{p}[i]\right\}=\operatorname{tr}\left(\tilde{\boldsymbol{S}}\left(\boldsymbol{H}_{c} \tilde{\boldsymbol{S}}\right)^{-1} \boldsymbol{A}^{2}\left(\boldsymbol{H}_{c} \tilde{\boldsymbol{S}}\right)^{-H} \tilde{\boldsymbol{S}}^{H}\right) \\
& =\operatorname{tr}\left(\boldsymbol{U}_{\tilde{s}} \boldsymbol{\Sigma}_{\tilde{s}} \boldsymbol{V}_{\tilde{s}}^{H}(\boldsymbol{U}_{c} \underbrace{\boldsymbol{\Sigma}_{c} \boldsymbol{V}_{c}^{H} \boldsymbol{U}_{\tilde{s}} \boldsymbol{\Sigma}_{\tilde{s}}}_{\boldsymbol{T}} \boldsymbol{V}_{s}^{H})^{-1} \boldsymbol{A}^{2}\left(\boldsymbol{U}_{c} \boldsymbol{\Sigma}_{c} \boldsymbol{V}_{c}^{H} \boldsymbol{U}_{\tilde{s}} \boldsymbol{\Sigma}_{\tilde{s}} \boldsymbol{V}_{\tilde{s}}^{H}\right)^{-H} \boldsymbol{V}_{\tilde{s}} \boldsymbol{\Sigma}_{\tilde{s}}^{H} \boldsymbol{U}_{\tilde{s}}^{H}\right) \\
& =\operatorname{tr}\left(\boldsymbol{\Sigma}_{\tilde{s}} \boldsymbol{V}_{\tilde{s}}^{H} \boldsymbol{V}_{\tilde{s}} \boldsymbol{T}^{-1} \boldsymbol{U}_{c}^{H} \boldsymbol{A}^{2} \boldsymbol{U}_{c} \boldsymbol{T}^{-H} \boldsymbol{V}_{\tilde{s}}^{H} \boldsymbol{V}_{\tilde{s}} \boldsymbol{\Sigma}_{\tilde{s}}^{H}\right) \\
& =\operatorname{tr}\left(\boldsymbol{\Sigma}_{\tilde{s}} \boldsymbol{T}^{-1} \boldsymbol{A}^{2} \boldsymbol{T}^{-H} \boldsymbol{\Sigma}_{\tilde{s}}^{H}\right)=\operatorname{tr}\left(\boldsymbol{A}^{2} \boldsymbol{\Sigma}_{\tilde{s}}^{2} \boldsymbol{T}^{-1} \boldsymbol{T}^{-H}\right),
\end{aligned}
$$

$\left\{\boldsymbol{u}_{\tilde{s}, 1}, \ldots, \boldsymbol{u}_{\tilde{s}, K}\right\}$ as the first $K$ columns of $\boldsymbol{V}_{c}$ and $\boldsymbol{U}_{\tilde{s}}$, respectively, we have $[\boldsymbol{C}]_{i j}=\left\langle\boldsymbol{v}_{c, i}, \boldsymbol{u}_{\tilde{s}, j}\right\rangle, i, j=1, \ldots, K$. Next we show that the eigenvalues of $\boldsymbol{C}$ denoted as $\phi_{i}, i=$ $1, . ., K$, always satisfy $\left|\phi_{i}\right| \leq 1$.

Denote $\left\{\boldsymbol{e}_{1}, \ldots, \boldsymbol{e}_{K}\right\}$ as the orthogonal basis of the $K$ dimensional space. Then the $l$-th component of the $C$ transform of the $j$-th basis is given by $\left[\boldsymbol{e}_{j}^{\prime}\right]_{l}=\left[\boldsymbol{C} \boldsymbol{e}_{j}\right]_{l}=[\boldsymbol{C}]_{l, j}=$ $\left\langle\boldsymbol{v}_{c, l}, \boldsymbol{u}_{\tilde{s}, j}\right\rangle$, where $\langle\cdot, \cdot\rangle$ denotes the inner product. Hence $\left\|\boldsymbol{e}_{j}^{\prime}\right\|^{2}=\sum_{l=1}^{K}\left|\left\langle\boldsymbol{v}_{c, l}, \boldsymbol{u}_{\tilde{s}, j}\right\rangle\right|^{2}$. Notice that since $\boldsymbol{V}_{c}$ and $\boldsymbol{U}_{\tilde{s}} \in$ $\mathrm{SU}(N)$ (i.e., special unitary group), then $\boldsymbol{V}_{c}^{H} \boldsymbol{U}_{\tilde{s}}$ also belongs to the $\mathrm{SU}(N)$; and therefore the $L_{2}$ norm of each column vector of the $N \times N$ matrix $\boldsymbol{V}_{c}^{H} \boldsymbol{U}_{\tilde{s}}$ equals to one, i.e., $\sum_{l=1}^{N}\left|\left\langle\boldsymbol{v}_{c, l}, \boldsymbol{u}_{\tilde{s}, j}\right\rangle\right|^{2}=1, j=1, \ldots, N$. Since $N \geq K$, we have $\left\|\boldsymbol{e}_{j}^{\prime}\right\|^{2}=\sum_{l=1}^{K}\left|\left\langle\boldsymbol{v}_{c, l}, \boldsymbol{u}_{\tilde{s}, j}\right\rangle\right|^{2} \leq 1, j=1, \ldots, K$. This is, the $L_{2}$ norm of the transformation by $\boldsymbol{C}$ of every basis vector is always less or equal to 1 . Every vector in the $K$-dimensional space can be written as a linear combination of the basis and therefore, the $C$ transform applied to any vector reduces the norm. In particular, it reduces the norm of the eigenvectors of $\boldsymbol{C}$. Therefore, we conclude that the eigenvalues of $\boldsymbol{C}$ satisfy $\left|\phi_{i}\right| \leq 1, \forall i$.

Substituting $\boldsymbol{T}^{-1}=\left[\boldsymbol{\Sigma}_{\tilde{s}}^{(t)}\right]^{-1} \boldsymbol{C}^{-1}\left[\boldsymbol{\Sigma}_{c}^{(t)}\right]^{-1}$ and the eigenvalue decomposition of $\boldsymbol{C}=\boldsymbol{W} \boldsymbol{\Phi} \boldsymbol{W}^{-1}$ (where $\boldsymbol{\Phi}=$ $\left.\operatorname{diag}\left(\phi_{1}, \ldots, \phi_{K}\right)\right)$ in (13) we obtain

$$
\begin{aligned}
P_{T} & =\operatorname{tr}\left(\boldsymbol{A}^{2} \boldsymbol{\Sigma}_{\tilde{s}}^{2} \boldsymbol{T}^{-1} \boldsymbol{T}^{-H}\right)=\operatorname{tr}\left(\boldsymbol{A}^{2} \boldsymbol{C}^{-1} \boldsymbol{C}^{-H} \boldsymbol{\Sigma}_{c}^{-2}\right) \\
& =\operatorname{tr}\left(\boldsymbol{A}^{2} \boldsymbol{\Phi}^{-1} \boldsymbol{\Phi}^{-H} \boldsymbol{\Sigma}_{c}^{-2}\right) \\
& =\sum_{i=1}^{K} A_{i}^{2} \lambda_{c, i}^{-2}\left|\phi_{i}\right|^{-2} \geq \sum_{i=1}^{K} A_{i}^{2} \lambda_{c, i}^{-2} .
\end{aligned}
$$

Denote the thin SVD of $\tilde{\boldsymbol{S}}=\boldsymbol{U}_{\tilde{s}}^{(t)} \boldsymbol{\Sigma}_{\tilde{s}}^{(t)} \boldsymbol{V}_{\tilde{s}}^{(t)}$. Finally, with $\tilde{\boldsymbol{S}}^{*}=\boldsymbol{H}_{c}^{H}$, the thin SVD decomposition becomes $\tilde{\boldsymbol{S}}^{*}=$ $\left(\boldsymbol{U}_{c}^{(t)} \boldsymbol{\Sigma}_{c}^{(t)} \boldsymbol{V}_{c}^{(t) H}\right)^{H}=\boldsymbol{V}_{c}^{(t)} \boldsymbol{\Sigma}_{c}^{(t) H} \boldsymbol{U}_{c}^{(t) H}$, i.e., $\boldsymbol{U}_{\tilde{s}}^{(t)}=\boldsymbol{V}_{c}^{(t)}$. Therefore $\boldsymbol{C}=\boldsymbol{V}_{c}^{(t) H} \boldsymbol{U}_{\tilde{s}}^{(t)}=\boldsymbol{I}_{K}$, and $\boldsymbol{C}$ has unit eigenvalues. Hence we have equality in (14) and $\tilde{\boldsymbol{S}}^{*}=\boldsymbol{H}_{c}^{H}$ is an optimal spreading matrix for linear MMSE precoding.

Remark: There are many other forms of the optimal spreading matrix $\tilde{\boldsymbol{S}}^{*}$ such that $\boldsymbol{C}=\boldsymbol{V}_{c}^{(t) H} \boldsymbol{U}_{\tilde{s}}^{(t)}$ has unit eigenvalues. Specifically, we need to construct an $N \times N$ matrix $\boldsymbol{U}_{\tilde{s}}$ that rotates the first $K$ columns vectors of $\boldsymbol{V}_{c}$ in the same $K$-dimensional subspace and keep invariant the $N-K$ dimensional subspace spanned by the $N-K$ remaining vectors. Consider first the real case. The constraints on the $K$ first columns of $\boldsymbol{U}_{\tilde{s}}$ are: (a) $\sum_{l=1}^{K}\left|\left\langle\boldsymbol{v}_{c, l}, \boldsymbol{u}_{\tilde{s}, j}\right\rangle\right|^{2}=1, j=1, \ldots, K$. [K equations.] (b) $\left\langle\boldsymbol{u}_{\tilde{s}, i}, \boldsymbol{v}_{c, m}\right\rangle=0, i=1, \ldots, K ; m=K+$ $1, \ldots, N$. [K. $(N-K)$ equations.] (c) $\left\langle\boldsymbol{u}_{\tilde{s}, i}, \boldsymbol{u}_{\tilde{r}, j}\right\rangle=\delta_{i j}, i, j=$ $1, \ldots, K .[(K-1)+(K-2)+\ldots+(K-K+1)+(K-K)=$ $K^{2}-\frac{1}{2} K(K+1)$ equations.] To construct $\boldsymbol{U}_{\tilde{s}}$, there are
$N K$ variables in the $K$ first columns of $\boldsymbol{U}_{\tilde{s}}$. After subtracting the number of constraints, we have $\left(K^{2}-K\right) / 2$ degrees of freedom, which is nothing more than the dimension of the $\mathrm{O}(K)$ (i.e., orthogonal group) as expected. In the complex case, there are $2 N K$ variables in the first $K$ columns of $\boldsymbol{U}_{\tilde{s}}$ and it can be shown that the solution generalizes to $\left(K^{2}-1\right)$ degrees of freedom that is the number of free parameters of the $\mathrm{SU}(K)$. To summarize, to construct the optimal spreading matrix with SVD decomposition $\tilde{\boldsymbol{S}}=\boldsymbol{U}_{\tilde{s}} \boldsymbol{\Sigma}_{\tilde{s}} \boldsymbol{V}_{\tilde{s}}^{H}$, we only have to find the unitary matrix $\boldsymbol{U}_{\tilde{s}}$ satisfying the above constraints on its $K$ first column vectors (i.e., range of $\tilde{\boldsymbol{S}}$ ). Moreover, there are $\left(K^{2}-1\right)$ degrees of freedom to select it.

\section{B. Chip-Wise Linear Precoding}

In chip-wise precoding, we do not explicitly use any spreading matrix at the transmitter. This is, the precoder takes $K$ symbols and outputs the spread vector of length $N$. Hence the spreading and precoding operations are effectively combined. The received signal at the $k$ th receiver is given by

$$
\boldsymbol{r}_{k}[i]=\boldsymbol{F}_{k} \boldsymbol{A} \boldsymbol{M}_{c} \boldsymbol{b}[i]+\boldsymbol{n}_{k}[i],
$$

where $\boldsymbol{M}_{c} \in \mathbb{C}^{N \times K}$ is the chip-wise precoding matrix. At each receiver $k$, the matched-filter $\boldsymbol{s}_{k}$ is applied to $\boldsymbol{r}_{k}[i]$. By stacking the outputs of all $K$ matched-filters we obtain

$$
\underbrace{\left[\begin{array}{c}
\boldsymbol{s}_{1}^{H} \boldsymbol{r}_{1}[i] \\
\boldsymbol{s}_{2}^{H} \boldsymbol{r}_{2}[i] \\
\vdots \\
\boldsymbol{s}_{K}^{H} \boldsymbol{r}_{K}[i]
\end{array}\right]}_{\boldsymbol{y}[i]}=\underbrace{\left[\begin{array}{c}
\boldsymbol{s}_{1}^{H} \boldsymbol{F}_{1} \\
\boldsymbol{s}_{2}^{H} \boldsymbol{F}_{2} \\
\vdots \\
\boldsymbol{s}_{K}^{H} \boldsymbol{F}_{K}
\end{array}\right]}_{\boldsymbol{H}_{c}} \boldsymbol{M}_{c} \boldsymbol{A b}[i]+\underbrace{\left[\begin{array}{c}
\boldsymbol{s}_{1}^{H} \boldsymbol{n}_{1}[i] \\
\boldsymbol{s}_{2}^{H} \boldsymbol{n}_{2}[i] \\
\vdots \\
\boldsymbol{s}_{K}^{H} \boldsymbol{n}_{K}[i]
\end{array}\right]}_{\boldsymbol{v}[i]}
$$

Differently from the bit-wise system model, here the channel matrix $\boldsymbol{H}_{c}$ is not a square matrix but has dimension $K \times N$ with $N \geq K$.

Chip-wise MMSE Precoding: Using an argument similar to [10], the linear MMSE chip-wise precoder is given by

$$
\boldsymbol{M}_{c}=\boldsymbol{H}_{c}^{\dagger}=\boldsymbol{H}_{c}^{H}\left(\boldsymbol{H}_{c} \boldsymbol{H}_{c}^{H}\right)^{-1} .
$$

It is easily seen that the SINR for each user is given by

$$
\operatorname{SINR}_{k}=\frac{A_{k}^{2}}{\sigma^{2}}, \quad k=1, \ldots, K .
$$

As before, if we assume that the required SINR for user $k$ is $\gamma_{k}$, the required power assigned to the $k$-th user becomes $A_{k}^{2}=\sigma^{2} \gamma_{k}$. Due to the precoding matrix, the required total transmit power becomes

$$
P_{T}=\operatorname{tr}\left(\boldsymbol{H}_{c}^{\dagger} \boldsymbol{A}^{2} \boldsymbol{H}_{c}^{\dagger H}\right)=\operatorname{tr}\left(\boldsymbol{A}^{2}\left(\boldsymbol{H}_{c} \boldsymbol{H}_{c}^{H}\right)^{-1}\right) .
$$


Remark: Note that under a fixed transmit power budget $P_{T}$, the linear MMSE precoder is given by $\boldsymbol{M}_{c}=\beta \boldsymbol{H}_{c}^{\dagger}$ with $\beta=\sqrt{P_{T} / \operatorname{tr}\left(\boldsymbol{A}^{2}\left(\boldsymbol{H}_{c} \boldsymbol{H}_{c}^{H}\right)^{-1}\right)}$ and $\operatorname{SINR}_{k}=\frac{\left(\beta A_{k}\right)^{2}}{\sigma^{2}}$.

Proposition 2: The above chip-wise linear MMSE precoding method is equivalent to the bit-wise linear MMSE precoding method with the optimal spreading matrix at the transmitter $\tilde{\boldsymbol{S}}^{*}$.

Proof: Using the SVD of $\boldsymbol{H}_{c}=\boldsymbol{U}_{c} \boldsymbol{\Sigma}_{c} \boldsymbol{V}_{c}^{H}$, the total transmit power required in the linear MMSE chip-wise precoder is given by

$$
\begin{aligned}
P_{T} & =\operatorname{tr}\left(\boldsymbol{H}_{c}^{\dagger} \boldsymbol{A}^{2} \boldsymbol{H}_{c}^{\dagger H}\right)=\operatorname{tr}\left(\boldsymbol{V}_{c} \boldsymbol{\Sigma}_{c}^{-1} \boldsymbol{U}_{c}^{H} \boldsymbol{A}^{2} \boldsymbol{U}_{s} \boldsymbol{\Sigma}_{c}^{-1} \boldsymbol{V}_{c}^{H}\right) \\
& =\operatorname{tr}\left(\boldsymbol{A}^{2} \boldsymbol{\Sigma}_{c}^{-2}\right)=\sum_{i=1}^{K} A_{i}^{2} \lambda_{c, i}^{-2} .
\end{aligned}
$$

Hence the transmit power with the chip-wise linear MMSE precoder is equal to the minimum transmit power in the bitwise solution given in (12).

Remark: The above result shows that it is not necessary to optimize the spreading operation at the transmitter. That is, by applying the simple chip-wise precoding operation we can obtain the optimal performance.

Chip-wise Wiener Precoding: The Wiener precoder given in (10) can be used in our chip-wise scheme by subsituting $\boldsymbol{H}_{b}$ by $\boldsymbol{H}_{c}$, resulting in the precoding matrix $\boldsymbol{M}_{c} \in \mathbb{C}^{N \times K}$. Next we propose a power loading algorithm that can be applied to both the bit-wise and chip-wise Wiener precoders. Consider the signal model (16). Define $\boldsymbol{G}=\boldsymbol{H}_{c} \boldsymbol{M}_{c}$. Then we can write $y_{k}[i]=A_{k} \boldsymbol{G}_{k k} b_{k}[i]+\sum_{i=1, i \neq k}^{K} A_{i} \boldsymbol{G}_{k i} b_{i}[i]+v_{k}[i], k=$ $1, \ldots, K$. In the Wiener precoder $\boldsymbol{M}_{c}$ is not the pseudo-inverse of $\boldsymbol{H}_{c}$ and therefore $\boldsymbol{G}$ is not a diagonal matrix. Hence, for a fixed loading matrix $\boldsymbol{A}$, the received SINR is given by

$$
\operatorname{SINR}_{k}=\frac{A_{k}^{2}\left\|\boldsymbol{G}_{k k}\right\|^{2}}{\sigma^{2}+\sum_{i=1, i \neq k}^{K} A_{i}^{2}\left\|\boldsymbol{G}_{k i}\right\|^{2}} .
$$

To achieve the target SINR $\gamma_{k}$ for each user $k$, we need to find the optimal powers $A_{k}^{2}, k=1, \ldots, K$. Now, different from the linear MMSE precoding, the power allocation problem is coupled with the problem of finding the optimal precoding matrix. Following the ideas of [11], in Algorithm 2 we propose the following iterative algorithm to solve the joint problem. In the algorithm we first fix the power loading values $\boldsymbol{A}(n)$ to find the precoding matrix and then, based on the precoding matrix, the power loading values are updated. Simulations show that the algorithm converges in about two or three iterations.

\section{Simulation Results}

Chip-wise precoding with matched-filter vs. bit-wise precoding with RAKE receiver: The bit-wise linear MMSE precoding with a RAKE receiver was proposed in [13]. The difference with the linear MMSE precoder considered in the Section III-A is that the receiver must also estimate the channel and apply a RAKE receiver, consequently, increasing the number of pilot symbols and the complexity of the receiver. We discuss this method only for comparison since we seek precoding solutions with simple receivers with no receiver

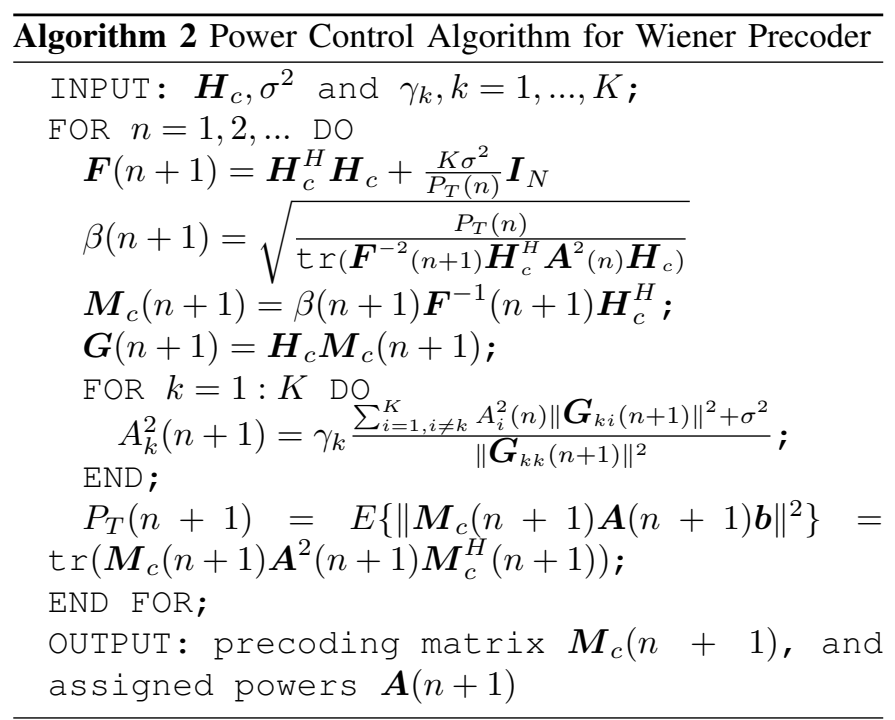

CSI. The RAKE receiver can be implemented with a matched filter using the effective spreading sequence (i.e., the $k$-th effective spreading sequence is $\overline{\boldsymbol{s}}_{k}=\boldsymbol{f}_{k} \star \boldsymbol{s}_{k}$ ) instead of the original spreading sequence. With our notation, the $k$ th effective spreading sequence is given by the convolution $\overline{\boldsymbol{s}}_{k}=\boldsymbol{F}_{k} \boldsymbol{S} \boldsymbol{e}_{k}=\boldsymbol{F}_{k} \boldsymbol{s}_{k}$, where we have limited the convolution to $N$ chip samples. Then, with the RAKE receiver the system model can be written as

$$
\begin{aligned}
\underbrace{\left[\begin{array}{c}
\boldsymbol{s}_{1}^{H} \boldsymbol{F}_{1}^{H} \boldsymbol{r}_{1}[i] \\
\boldsymbol{s}_{2}^{H} \boldsymbol{F}_{2}^{H} \boldsymbol{r}_{2}[i] \\
\vdots \\
\boldsymbol{s}_{K}^{H} \boldsymbol{F}_{K}^{H} \boldsymbol{r}_{K}[i]
\end{array}\right]}_{\boldsymbol{y}[i]}=\underbrace{\left[\begin{array}{c}
\boldsymbol{s}_{1}^{H} \boldsymbol{F}_{1}^{H} \boldsymbol{F}_{1} \boldsymbol{S} \\
\boldsymbol{s}_{1}^{H} \boldsymbol{F}_{2}^{H} \boldsymbol{F}_{2} \boldsymbol{S} \\
\vdots \\
\boldsymbol{s}_{K}^{H} \boldsymbol{F}_{K}^{H} \boldsymbol{F}_{K} \boldsymbol{S}
\end{array}\right]}_{\boldsymbol{H}_{b}} \boldsymbol{M}_{b} \boldsymbol{A b}[i] \\
+\underbrace{\left[\begin{array}{c}
\boldsymbol{s}_{1}^{H} \boldsymbol{F}_{1}^{H} \boldsymbol{n}_{1}[i] \\
\boldsymbol{s}_{2}^{H} \boldsymbol{F}_{2}^{H} \boldsymbol{n}_{2}[i] \\
\vdots \\
\boldsymbol{s}_{K}^{H} \boldsymbol{F}_{K}^{H} \boldsymbol{n}_{K}[i]
\end{array}\right]}_{\boldsymbol{v}[i]} .
\end{aligned}
$$

It is easily seen that the linear MMSE precoding solution still yields $\boldsymbol{M}_{b}=\boldsymbol{H}_{b}^{-1}$, where $\boldsymbol{H}_{b}$ is defined in (22). The signal to noise ratio for user $k$ is

$$
\operatorname{SINR}_{k}=\frac{A_{k}^{2}}{\sigma^{2}\left\|\boldsymbol{F}_{k} \boldsymbol{s}_{k}\right\|^{2}}, \quad k=1, \ldots, K,
$$

and the required power to achieve an SINR value $\gamma_{k}$ becomes $A_{k}^{2}=\sigma^{2} \gamma_{k} \boldsymbol{s}_{k}^{H} \boldsymbol{F}_{k}^{H} \boldsymbol{F}_{k} \boldsymbol{s}_{k}$. Therefore, the total transmitted power is given by

$$
P_{T}=\mathbb{E}\left\{\left\|\boldsymbol{S} \boldsymbol{H}_{b}^{-1} \boldsymbol{A} \boldsymbol{b}[i]\right\|^{2}\right\}=\operatorname{tr}\left(\boldsymbol{S} \boldsymbol{H}_{b}^{-1} \boldsymbol{A}^{2} \boldsymbol{H}_{b}^{-H} \boldsymbol{S}^{H}\right) .
$$

Notice that the Wiener precoding solution can also be applied to the system in (22).

Next we compare the chip-wise linear MMSE precoder given in Section III-B (which is equivalent to the optimal bitwise linear MMSE precoder) with the above bit-wise precoder with a RAKE receiver. We assume that the target SINR per user is constant for all users, $\gamma_{k}=13 \mathrm{~dB}, k=1, \ldots, K$. We consider random codes and Gold codes with spreading gain 


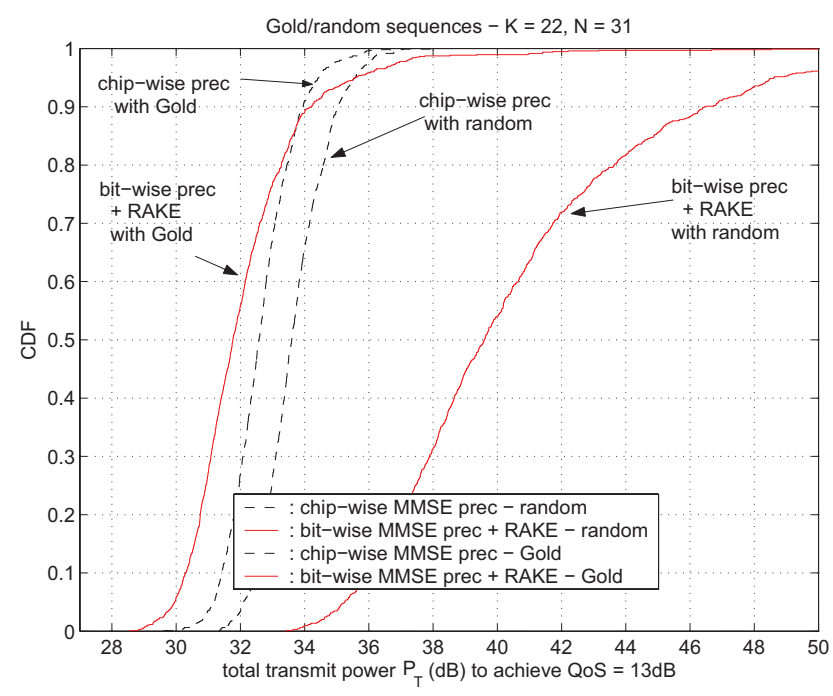

Fig. 1. Chip-wise precoding with matched-filter vs. bit-wise precoding with RAKE receiver: CDF of the required power $P_{T}$ at the transmitter to achieve $\gamma_{k}=13 \mathrm{~dB}, \forall k$. Spreading gain $N=31, K=22$ users.

$N=31$ and the total number of users $K=22$. We assume that each mobile user experiences an independent multipath channel $\boldsymbol{f}_{k}=\left[f_{k, 1}, \ldots, f_{k, L}\right]^{T}$ with $L=3$ resolvable paths, and the transmitter has perfect CSI of all users. The path gains are generated according to $f_{k, i} \sim \mathcal{N}_{c}\left(0, \frac{1}{L}\right)$. The results are averaged over 1000 different channel realizations. The cumulative distribution function (CDF) of the required power at the transmitter to achieve the minimum SINR at the receivers is shown in Fig. 1. With Gold sequences the RAKE receiver brings $0.5 \mathrm{~dB}$ gain on average (i.e., $\mathrm{CDF}=50 \%$ ) compared with the simple chip-wise precoder with matchedfilter receiver. Note that the performance of a communication system is dominated by the outage events. Given an outage probability $p_{\text {out }}$, we define the corresponding outage power $P_{\text {out }}$ as $p_{\text {out }}=\operatorname{Pr}\left\{P_{T} \geq P_{\text {out }}\right\}$. It is seen that although on average the RAKE receiver is slightly better, it is more prone to outage. For instance, consider in the plot the 5\% outage probability (i.e., $\mathrm{CDF}=95 \%$ ) for which the chip-wise precoder requires around $34.5 \mathrm{~dB}$ whereas the RAKE receiver requires around $35.5 \mathrm{~dB}$. When considering the $1 \%$ outage probability (i.e., $\mathrm{CDF}=99 \%$ ), this effect is more pronounced and the RAKE receiver requires $5 \mathrm{~dB}$ more than the chip-wise precoder to achieve the same performance. This effect will be more clear in the BER simulation results [cf. Fig. 5 and Fig. 6]. Interestingly, the performance of the precoder with RAKE receiver decays considerably when random sequences are used although it only has a minimum effect in performance of the chip-wise precoder. Therefore, the chip-wise precoder is not only simpler (and it makes the receiver simpler since no CSI is required at the receiver) but it also has excellent performance. From the above simulation results we can conclude that the bit-wise precoder with RAKE receiver makes the mobile units more complex and does not bring much improvements with Gold sequences and it can be very detrimental with random spreading sequences.

Linear precoding vs. linear MUD - total transmit power: Next we compare linear MUD with linear precoding assuming

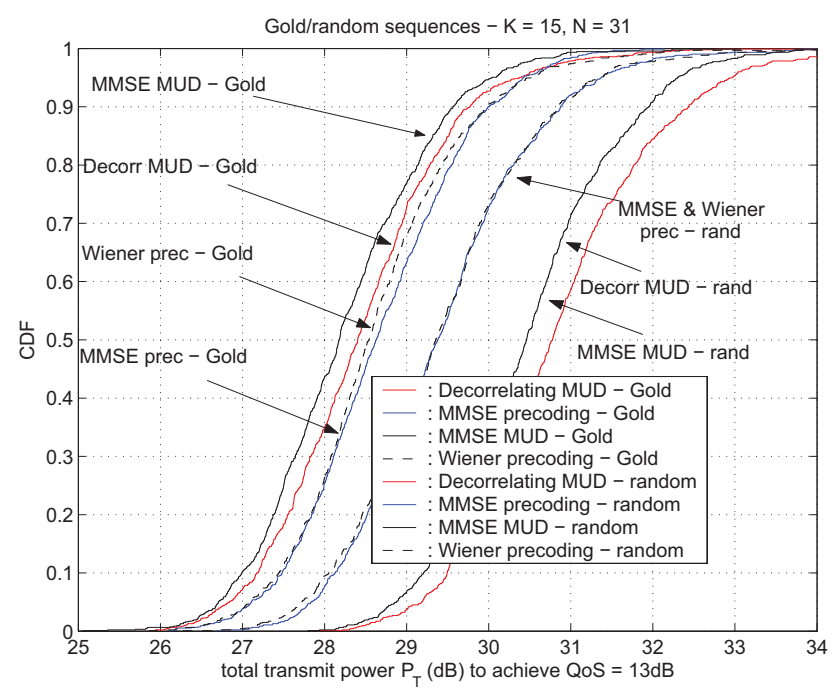

Fig. 2. Linear precoding vs. linear MUD: CDF of the required power $P_{T}$ at the transmitter to achieve $\gamma_{k}=13 \mathrm{~dB}, \forall k$. Spreading gain $N=31, K=15$ users.

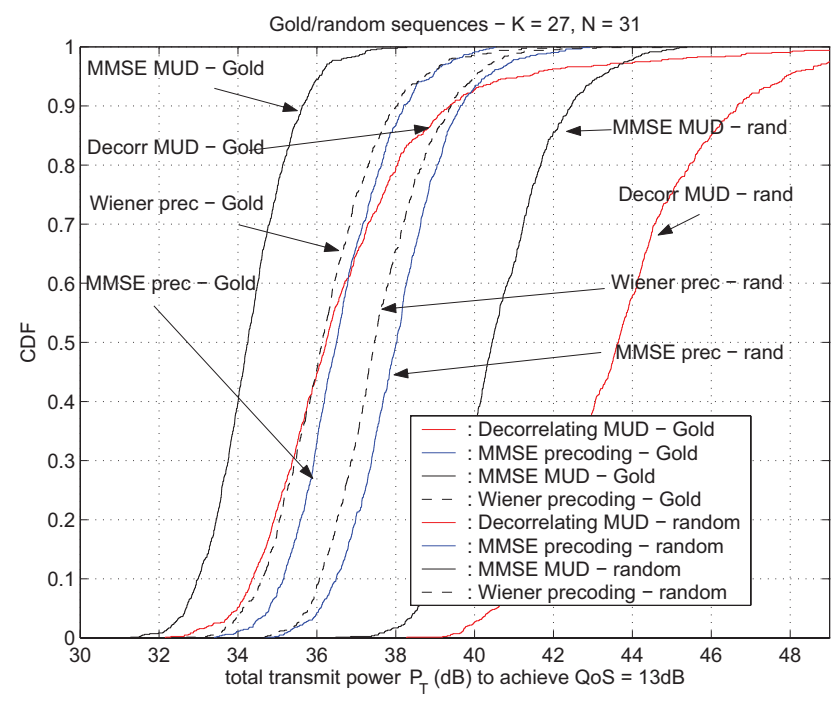

Fig. 3. Linear precoding vs. linear MUD: CDF of the required power $P_{T}$ at the transmitter to achieve $\gamma_{k}=10 \mathrm{~dB}, \forall k$. Spreading gain $N=31, K=27$ users.

similar simulations parameters. We compare the CDF of the required total power $P_{T}$ at the transmitter to achieve a target SINR $\gamma_{k}=13 \mathrm{~dB}, \forall k$, in each of the four following schemes: (a) linear decorrelating MUD [cf. Eq.(4)]; (b) linear MMSE MUD [cf. Alg. 1]; (c) chip-wise linear MMSE precoder, [cf. Eq.(19)]; and (d) chip-wise Wiener precoder [cf. Alg. 2]. Simulations are performed for spreading gain $N=31$, with Gold and random spreading sequences. Fig. 2 shows the results with $K=15$ users and Fig. 3 shows the results with $K=27$ users. It is seen that with Gold codes, MUD is slightly better (although only $0.5 \mathrm{~dB}$ of difference with linear precoding when 15 users are considered), whereas with random codes linear precoding largely outperforms MUD. Notice that the Wiener precoder is slightly better than the MMSE precoder. It is also seen that the total power required in the precoding solutions is almost independent of the chosen spreading sequences and therefore, an outage event is less 


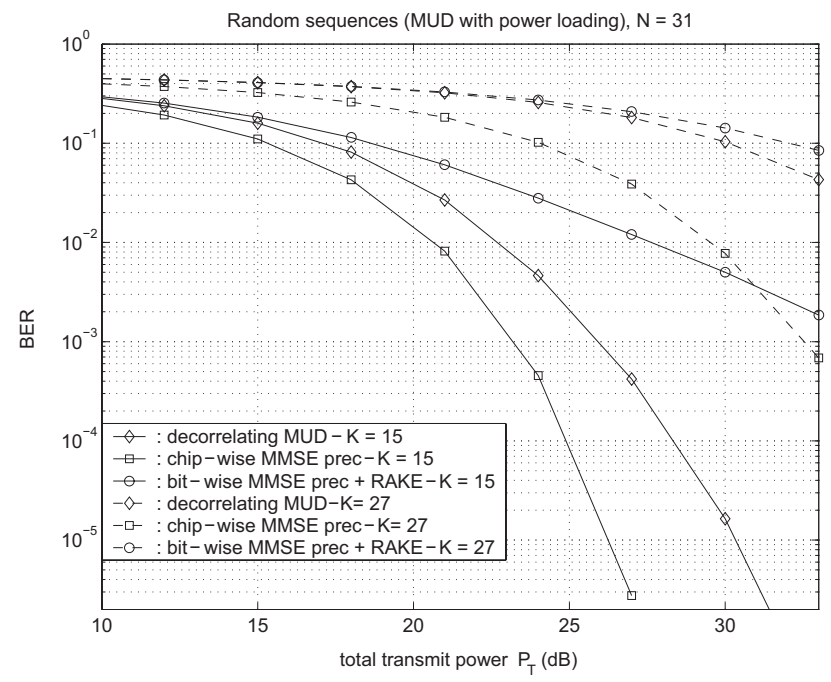

Fig. 4. Linear precoding vs. linear MUD: BER performance with random spreading sequences. Spreading gain $N=31, K=15$ and $K=27$ users.

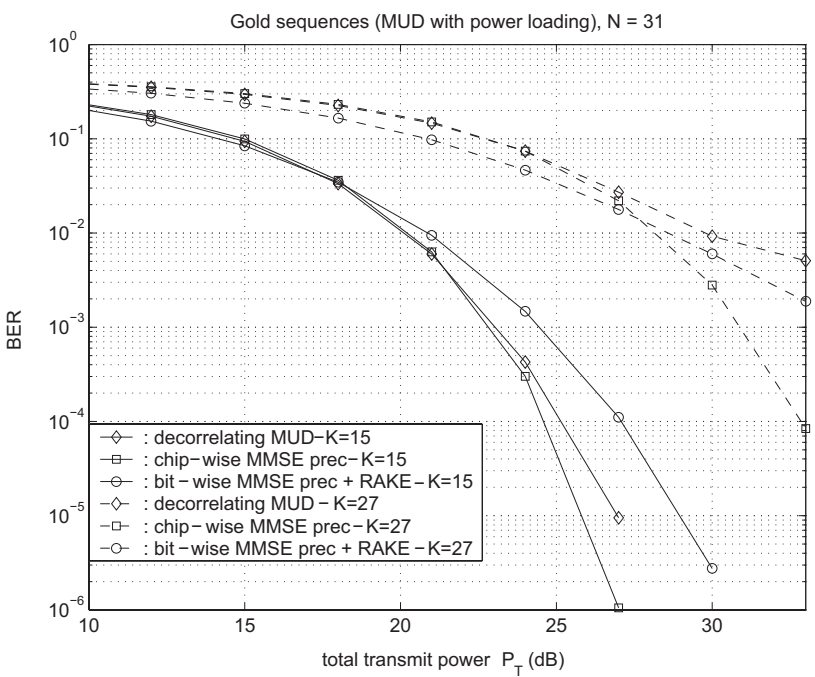

Fig. 5. Linear precoding vs. linear MUD: BER performance with Gold spreading sequences. Spreading gain $N=31, K=15$ and $K=27$ users.

likely to occur. Although the linear MMSE MUD solution seems to be quite effective with Gold codes, we recall that it is unlikely to be implemented in the downlinks of most wireless systems due to the amount of required feedback information to implement perfect power control and other issues discussed in Section I. Also notice that the linear decorrelator offers very poor performance in heavily loaded systems, which does not occur to the linear MMSE linear precoder.

Linear precoding vs. linear MUD - BER performance: Fig. 4 (random spreading sequences) and Fig. 5 (Gold spreading sequences) show the BER performance of the various linear MUD and linear precoding methods. The results are averaged over 100 channel realizations and QPSK modulation is employed. Recall that the linear MMSE precoder is equivalent to the transmitter counterpart of the decorrelator. For the decorrelating MUD we consider perfect power loading to achieve the same SNR across the users. It is seen that the linear MMSE precoder with RAKE only performs slightly better with Gold sequences in the very low SNR region. In all the other cases, the chip-wise linear MMSE precoder obtains much better results. On the other hand, the chip-wise MMSE precoder obtains much better results than the decorrelating MUD, especially in heavily loaded systems. These results are due to the outage events of the decorrelating MUD observed in Fig. 2 and Fig. 3. Again, it is seen that the BER performance of the chip-wise precoding solution is almost independent of the chosen spreading sequence.

\section{Conclusions}

In this work we have compared the performance of linear precoding and linear MUD in the downlink of TDD-CDMA systems. We have proposed different linear precoding schemes and our results reveal that precoding can outperform the more complex MUD. Moreover, we have shown that the proposed chip-wise linear MMSE precoding method is optimal in the sense that it requires the minimum total transmitted power to meet a certain receiver SINR target. These results strongly motivate the use of transmit precoding in the downlink of TDDCDMA systems due to the multiple advantages over MUD, including the simple implementation of power control and user scheduling, and the reduction of the power consumption and complexity at the mobile unit.

\section{REFERENCES}

[1] A. Duel-Hallen, S. Hu, and H. Hallen, "Long-range prediction of fading signals: Enabling adapting transmission for mobile radio channels," IEEE Signal Processing Mag., vol. 17, no. 3, pp. 62-75, May 2000.

[2] R. Esmailzadeh, E. Sourour, and M. Nakagawa, "Pre-rake diversity combining in time-division duplex CDMA mobile communications," IEEE Trans. Veh. Technol., vol. 48, pp. 795-801, May 1999.

[3] R. F. H. Fischer and C. A. Windpassinger, "Improved MIMO precoding for decentralized receivers resembling concepts from lattice reduction," in Proc. IEEE Globecom, Dec. 2003, vol. 4, pp. 1852-1856.

[4] R. A. Horn and C. R. Johnson, Matrix Analysis. Cambridge, U.K.: Cambridge University Press, 1985.

[5] M. Joham, K. Kusume, M. H. Gzara, and W. Utschick, "Transmit Wiener filter for the downlink of TDD DS-CDMA systems," in Proc. 7th Int. Symp. on Spread-Spectrum Tech. \& Apps., Sep. 2002, pp. 9-13.

[6] M. Joham, W. Utschick, and J. A. Nossek, "Linear transmit processing in MIMO communications systems," IEEE Trans. Signal Processing, vol. 53 , no. 8 , pp. $2700-2712$, Aug. 2005.

[7] X. Liu, E. K. P. Chong, and N. B. Shroff, "A framework for opportunistic scheduling in wireless networks," Computer Networks: International J. of Computer and Telecommun. Networking, vol. 41, no. 4, pp. 451-474, Mar. 2003.

[8] Y. Liu and E. Khightly, "Opportunistic fair scheduling over multiple wireless channels," in Proc. IEEE INFOCOM, Mar. 2003, vol. 2, pp. $1105-1115$

[9] C. Peel, B. Hochwald, and L. Swindlehurst, "A vector-perturbation technique for near-capacity multi-antenna multi-user communicationPart 2," IEEE Trans. Commun., submitted.

[10] D. Reynolds, A. Høst-Madsen, and X. Wang, "Adaptive transmitter precoding for time division duplex CDMA," in EURASIP J. Applied Sig. Proc., vol. 12, pp. 1325-1334, Dec. 2002.

[11] S. Ulukus and R. Yates, "Adaptive power control and MMSE interference suppression," Wireless Networks, vol. 4, no. 6, pp. 489-496, Nov. 1998.

[12] S. Verdú, Multiuser Detection. Cambridge, UK: Cambridge University Press, 1998.

[13] B. R. Vojcic and W. M. Jang, "Transmitter precoding in synchronous multiuser communications," IEEE Trans. Commun., vol. 46, no. 10, pp. 1346-1355, Oct. 1998.

[14] C. Windpassinger, R. F. H. Fischer, T. Vencel, and J. B. Huber, "Precoding in multi-antenna and multi-user communications," IEEE Trans. Wireless Commun., vol. 3, no. 4, pp. 1305-1316, July 2004. 